\title{
Associações entre a exposição a espaços verdes e o desenvolvimento de asma e doença alérgica em ambientes urbanos: Da coerência à controvérsia científica
}

\section{Associations between exposure to green spaces and the development of asthma and allergic disease in urban environments: From coherence to scientific controversy}

Carolina Dinis', Mariana Farraia ${ }^{2,3}$ (DD, Inês Paciência ${ }^{2}$ (D), Ana Isabel Ribeiro² ${ }^{\text {ID }}$, André Moreira $^{3}$ (D), Luís Delgado 3 iD, João Cavaleiro Rufo ${ }^{3}$ iD

' ESS - Escola Superior de Saúde, Politécnico do Porto, Porto, Portugal

${ }^{2}$ EPIUnit - Instituto de Saúde Pública, Universidade do Porto, Porto, Portugal

${ }^{3}$ Serviço de Imunologia Básica e Clínica, Departamento de Patologia, Faculdade de Medicina da Universidade do Porto,

Porto, Portugal

\section{RESUMO}

A exposição ao ambiente urbano tem sido associada ao desenvolvimento de asma e doença alérgica. No entanto, as associações encontradas ainda geram controvérsia, com alguns estudos a indicarem os espaços verdes como protetores no desenvolvimento das doenças alérgicas, enquanto outros apontam essas exposições como tendo um papel indutor na exacerbação da sintomatologia associada. O presente artigo pretende rever a evidência científica até aqui publicada, de forma a debater o eventual efeito da exposição a espaços verdes urbanos no desenvolvimento de asma e doença alérgica em crianças, com especial importância dada aos estudos realizados em Portugal.

Palavras-chave: Alergia, ambiente urbano, asma, espaços verdes. 


\section{ABSTRACT}

Exposure to urban environments has been associated with the development of asthma and allergic disease. However, the associations found still generate controversy, with some studies indicating green spaces as protectors for the development of allergic diseases, while others point out these exposures as having an inducing role in the exacerbation of the associated symptomatology. This article aims to review the scientific evidence published so far in order to discuss the possible effect of exposure to urban green spaces on the development of asthma and allergic disease in children, with special focus on studies conducted in Portugal.

Keywords: Allergy, asthma, green spaces, urban environment.

(C) 202I Sociedade Portuguesa de Alergologia e Imunologia Clínica. Published by Publicações Ciência \& Vida. This is an open access article under the CC BY-NC-ND license (http://creativecommons.org/licenses/by-nc-nd/4.0/).

\section{O QUE SÃO ESPAÇOS VERDES}

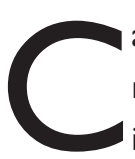
ada vez mais vivemos numa sociedade que valoriza o bem-estar e a saúde, onde a mesma é influenciada positiva ou negativamente, pela qualidade ambiental, ou seja, pela perceção estruturada dos elementos que constituem o meio envolvente ${ }^{1,2}$. Posto isto, aliado aos avanços tecnológicos das últimas décadas, a temática dos espaços verdes é cada vez mais objeto de estudo, existindo já evidências científicas que reforçam a importância destes espaços na saúde e longevidade das populações ${ }^{3}$.

Quando falamos em espaços verdes urbanos referimo-nos a todos os jardins, parques, ou a todas as áreas livres ocupados por vegetação num meio urbanol,4. A nível ambiental, estes espaços permitem a regularização da temperatura, a renovação do ar e o aumento da biodiversidade ${ }^{5}$. A nível social e cultural, a exposição a estes espaços parece estar associada a um aumento da interação social e a uma melhoria e equilíbrio do estado físico e psicológico daqueles que os frequentam, promovendo um aumento da qualidade de vida ${ }^{3,6}$. Porém, ao nível da patologia alérgica, existem informações contraditórias sobre o efeito da exposição a estes es- paços e o desenvolvimento de alergia e asma nas crianças, tendo sido identificados tanto como espaços protetores como também responsáveis pelo aumento da doença ${ }^{7}$. Deste modo, o presente artigo pretende abordar a evidência científica até aqui publicada, de forma a debater o eventual efeito da exposição a espaços verdes urbanos no desenvolvimento de asma e doença alérgica em crianças. Tendo em conta a novidade e o contexto, será dada especial relevância a estudos realizados em Portugal.

\section{MEDIÇÃO DA EXPOSIÇÃO A ESPAÇOS VERDES}

De forma a podermos determinar os impactos positivos e negativos da exposição a espaços verdes na saúde, é crucial torná-los quantificáveis e qualificáveis. Para tal, são utilizados sistemas de informação geográfica (SIG) e outras geotecnologias, como a deteção remota. Os SIG são sistemas computacionais desenvolvidos para recolher, armazenar, organizar, manipular e analisar informações geográficas ${ }^{8}$, como por exemplo as referentes à presença de vegetação e espaços verdes. 
A deteção remota é uma técnica usada para obter dados sobre as propriedades físicas, químicas e biológicas dos objetos sem que seja necessário entrar em contacto físico com elas, o que é conseguido graças a sensores presentes em aviões e/ou satélites que medem a quantidade de radiação eletromagnética que é refletida, emitida ou difundida por um dado objeto (ex. vegetação), permitindo depois identificá-lo com base na sua assinatura espectral.

O índice de vegetação por diferença normalizada é frequentemente utilizado para estimar a quantidade de vegetação numa determinada área através de imagens de satélite (por exemplo, provenientes do satélite Landsat ${ }^{9}$ e Sentinel-2 $\left.{ }^{10}\right)$. Este índice, normalmente abreviado para NDVI (Normalized difference vegetation index), tem como base a refletância da superfície terrestre dos comprimentos de onda vermelho visível (VISR) e infravermelho próximo (NIR) e é obtido através da equação abaixo (equação I):

$$
N D V I=\frac{N I R-V I S R}{N I R+V I S R} \quad \text { equação I }
$$

O princípio subjacente utilizado no cálculo do NDVI é que a clorofila na vegetação saudável absorve a radiação na região vermelha visível $(630-690 \mathrm{~nm})$ do espetro eletromagnético e reflete a radiação na região do infravermelho próximo (760-900 nm) e radiação na região verde visível (por isso é que os nossos olhos veem a vegetação com a cor verde). É um índice sem unidade que varia entre - I e I, com valores mais altos correspondendo a uma maior densidade de vegetação saudável ${ }^{\prime \prime}$. A título de exemplo, a Figura I ilustra a distribuição do NDVI para a área metropolitana do Porto.

Apesar do NDVI ser o índice de medição utilizado na grande maioria dos estudos, alguns autores recorrem a diferentes métodos de medição, de forma a quantificar o impacto destes espaços. De entre estes métodos encontram-se a medição da percentagem de espaço verde dentro do perímetro de residência, medição da distância entre a residência e espaço verde mais próximo e mesmo

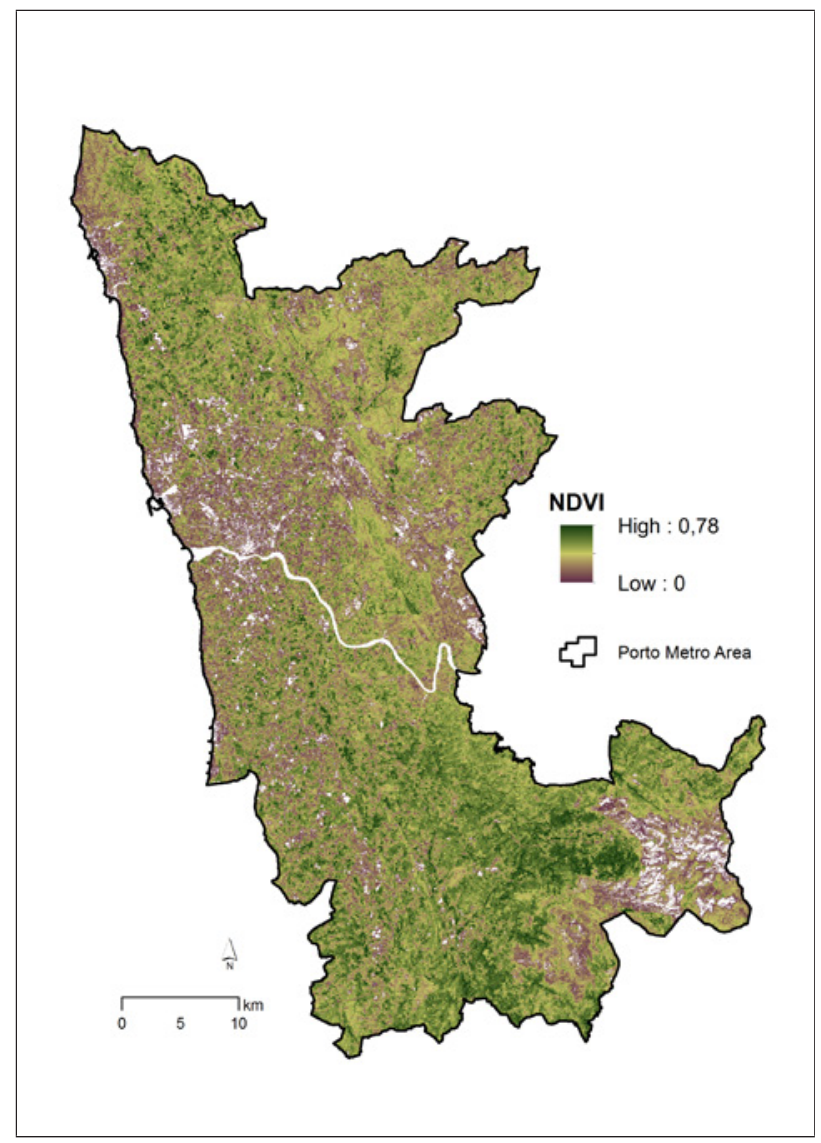

Figura I. Distribuição do NDVI pela área metropolitana do Porto

a percentagem de espaços verdes e parques, usando diferentes limiares de distância em torno da residência'2. Note-se que, para além destas, existem outras formas de medir a exposição a espaços verdes, sendo que o método de medição adotado vai sempre depender do objetivo do estudo em questão. Para além disto, no mesmo estudo é possível realizar-se uma combinação de múltiplas medições de exposição do espaço verde, como por exemplo a proximidade, a qualidade e a área desse mesmo espaço ${ }^{12}$. Convém também clarificar que este tipo de análise pode ser adaptado para qualquer espaço de exposição, como por exemplo escolas. Esta diversidade metodológica deve ser tida em conta na interpretação de resultados, especialmente na comparação entre diferentes estudos. 


\section{ÁREAS VERDES NO DESENVOLVIMENTO DE DOENÇA ALÉRGICA}

A Revolução Industrial, no século XVIII, trouxe enormes transformações no cenário urbano europeu, levando a um aumento da área construída, reduzindo assim o número de áreas verdes. Em consequência, o aumento destas áreas levou a diversas mudanças no estilo de vida das populações, como por exemplo a diminuição da prática de exercício físicol,3,12.

Estudos epidemiológicos recentes sugerem que o acesso a espaços verdes em áreas urbanas pode ter um impacto positivo na saúde e bem-estar da população, tanto a nível físico como mental ${ }^{13}$. No mesmo sentido, alguns estudos publicados colocam como hipótese que a exposição a áreas verdes pode ter um impacto significativo no desenvolvimento de doença alérgica e asma nas crianças $^{4,14-19}$. Esta hipótese tem por base três argumentos. Primeiro, o efeito modelador da exposição a um ambiente mais natural e biodiversificado no sistema imune, podendo promover a tolerância a alergénios ${ }^{20-24}$. Segundo, alguns espaços verdes urbanos, especialmente os constituídos por vegetação arbórea, podem funcionar como barreira protetora contra poluentes do ar que caracterizam uma zona urbana, reduzindo assim o efeito destes poluentes na exacerbação de sintomas de asma ou na promoção do stresse oxidativo ${ }^{25}$. E terceiro, estas áreas verdes podem ter um efeito reparador a nível psicossocial para as crianças, tal como demonstrado por Chen et al., num estudo em crianças com asma (diagnóstico clínico) com idades compreendidas entre os 9 e os 17 anos, onde as interações entre a exposição a espaços verdes e complicações na relação familiar apresentaram efeitos no controlo da doença, perda de função pulmonar e inflamação das vias aéreas ${ }^{26}$.

No entanto, como demonstrado numa revisão sistemática realizada por Cavaleiro Rufo et al. ${ }^{7}$, o efeito da exposição a espaços verdes urbanos no desenvolvimento de doença alérgica e asma nas crianças é bastante controverso, sendo apontado como tendo efeito protetor em alguns estudos, como fator de risco noutros, ou simplesmente como não tendo qualquer relação. A justificação para esta discrepância prende-se principalmente com diferenças entre as regiões dos estudos e a tipologia da vegetação. Por exemplo, um estudo realizado em Espanha por Tischer et al. mostrou que o efeito da exposição a áreas verdes varia de forma significativa entre as regiões eurossiberianas e mediterrâneas do país, no caso da primeira as áreas verdes foram associadas a uma menor prevalência de pieira, enquanto na segunda estes espaços mostraram estar mais associados a uma menor prevalência de bronquite, mas não de sibilância ${ }^{19}$.

Uma razão para a associação positiva entre o desenvolvimento de doença e a exposição a espaços verdes pode basear-se na mesma biodiversidade que mencionamos como fator protetor. Por outro lado, espaços verdes significam, de forma geral, mais aeroalergénios originados na fauna e flora que caracterizam estas áreas, podendo assim contribuir para o aumento de exacerbações dos sintomas em indivíduos sensibilizados ${ }^{27}$. Por exemplo, na cidade espanhola de Sabadell, Dadvand et al. constataram que as crianças que viviam perto de florestas tinham maior risco de desenvolver rinoconjuntivite alérgica (OR [95\% IC]: I,27 [0,94; I,70]) $)^{15}$. Noutro estudo, a mesma proximidade a áreas verdes foi associada a um risco acrescido de $27 \%$ no desenvolvimento de sensibilização atópica em crianças com 7 anos de idade (OR [95\% IC]: 1,27 [I,02; I,58]) ${ }^{27}$.

Tendo em conta estas diferenças, é importante olharmos em concreto para a investigação realizada em Portugal no estudo de associações entre a exposição a espaços verdes e o desenvolvimento de doença alérgica e asma em crianças.

\section{O EFEITO DAS ÁREAS VERDES NAS DOENÇAS ALÉRGICAS EM PORTUGAL}

A prevalência de asma e doenças alérgicas em Portugal é elevada, tendo Sa-Sousa et al. ${ }^{28}$, num estudo publi- 
cado em 2012, demonstrado uma prevalência de 6,8\% de asma em Portugal, e Morais-Almeida et al. ${ }^{29}$, em 2013, uma prevalência de rinite de $43,4 \%$. Tendo em conta estes números, e considerando que a urbanização do território é um fator em constante crescimento, é importante perceber o impacto das áreas verdes em ambiente urbano e de que forma estes espaços estão associados com a prevalência destas patologias em território português.

Existem vários estudos publicados em Portugal focados na associação entre os espaços verdes e a saúde das crianças em meio urbano. Em 2017, Magalhães et al. publicaram um estudo em que a atividade física de 969 adolescentes, pertencentes à coorte portuguesa EPITeen, foi relacionada com a presença de espaços verdes em redor da habitação ${ }^{30}$. Porém, não foram encontradas quaisquer associações entre estas duas variáveis, o que denota que a presença de espaços verdes na proximidade do local de residência não influencia significativamente a prática de exercício físico nos adolescentes. No sentido contrário, um estudo realizado no município de Lisboa concluiu que crianças que vivem em bairros mais recentes e com mais espaços verdes estão mais protegidas contra a obesida$d^{31}$. Convém frisar que esta diminuição na prevalência de obesidade pode não estar associada à prática de exercício físico, mas a uma menor concentração de poluentes com capacidade de disrupção endócrina nestes bairros ${ }^{32}$, justificando assim as observações suprarreferidas. Por outro lado, este efeito da exposição a áreas verdes na prevenção da obesidade não se verificou num outro estudo, mais recente, que teve como amostra a coorte portuguesa Geração XXI, que engloba mais de $\mathbf{8 0 0 0}$ participantes ${ }^{33}$. Neste estudo, a exposição a espaços verdes (medida por NDVI e distância pedonal a parques e jardins) não se associou de forma significativa com a prevalência de obesidade aos 7 anos, contrariando assim os resultados observados pelo estudo realizado em Lisboa ${ }^{33}$. Poderão as diferenças entre regiões justificar a inconsistência entre estes resultados?

Outro estudo realizado em Portugal ${ }^{34}$ investigou as relações entre a presença de espaços verdes ao redor de escolas primárias e o estado de saúde das crianças, tendo sido identificadas associações positivas entre a exposição a espaços verdes e o aumento da função pulmonar. Segundo os autores, estas associações podem ser justificadas pelo efeito calmante (soothing) das áreas verdes na atividade parassimpática do sistema nervoso autóno$\mathrm{mo}^{34}$. Este efeito protetor das áreas verdes nas proximidades do recinto escolar também foi verificado num outro estudo, realizado por Ribeiro et al. ${ }^{35}$, neste caso para a redução da carga alostática em crianças da coorte Geração XXI. Neste estudo, crianças a frequentar escolas com espaços verdes num raio de $\mathbf{8 0 0}$ metros apresentavam uma menor carga alostática (redução média de aproximadamente $30 \%$ ) e, por cada quilómetro de distância para o espaço verde mais próximo, a carga alostática aumentava em cerca de $12 \%$. Curiosamente, o mesmo efeito não se verificou para a exposição a espaços verdes ao redor das habitações dos participantes ${ }^{35}$.

No entanto, só muito recentemente foi publicado um estudo longitudinal, conduzido em Portugal, em que se estudaram as associações entre a exposição a espaços verdes e o desenvolvimento de doença alérgica e asma ${ }^{36}$. Este estudo analisou 1050 crianças pertencentes à coorte Geração XXI que tiveram como primeiro local de habitação uma residência localizada no município do Porto. Foi estimado um valor de NDVI nos 100 metros circundantes a cada habitação, cujo efeito no desenvolvimento de doença alérgica, asma, e sintomatologia associada, foi estudado por regressão logística. Os resultados demonstraram que uma maior exposição a espaços verdes ao redor da primeira habitação da criança está associado a uma menor prevalência de asma e rinite alérgica aos 7 anos (OR 95\% IC : 0,4I [0,18:0,97] e 0,37 $[0,15: 0,93]$, respetivamente) ${ }^{36}$.

Finalmente, um outro estudo desenvolvido em Portugal avaliou a associação entre a presença de árvores e o seu tipo de folhagem, nomeadamente árvores de folha larga e coníferas, considerando um raio de 500 metros em torno das escolas, e a inflamação das vias áreas em crianças $^{37}$. Os autores observaram que a presença de 
árvores em redor das escolas está associada a níveis de óxido nítrico mais baixos, sugerindo a existência de um efeito benéfico da presença de árvores na diminuição da inflamação das vias aéreas ${ }^{37}$.

\section{CONCLUSÕES}

O efeito da exposição a espaços verdes no desenvolvimento de doença alérgica e asma é ainda bastante controverso, parecendo variar entre regiões e possivelmente dependendo da biodiversidade dos espaços e do comportamento da população exposta. Para além disso, apesar do NDVI ser o índice de medição utilizado na grande maioria dos estudos, alguns autores recorrem a diferentes métodos de medição, de forma a quantificar o impacto destes espaços. Convém também clarificar que as observações podem ser influenciadas pelo local de exposição, embora nestes casos apenas residências e escolas tenham sido objeto de estudo. Esta diversidade metodológica deve ser tida em conta na interpretação de resultados.

Contudo, os estudos realizados em Portugal sugerem que existe um efeito protetor da exposição às áreas verdes, quer em ambiente escolar quer em ambiente residencial, no desenvolvimento de sensibilização alérgica nas crianças. É importante ter em conta o contexto regional destes estudos, com a maioria a serem realizados em Lisboa e Porto, zonas densamente urbanizadas.

Tendo isto em conta, enaltece-se a necessidade de perceber o efeito modelador da região em estudo nas associações entre os espaços verdes e desenvolvimento de doença, com especial atenção para as comparações entre os meios urbanos e os meios rurais, que diferem não só em termos de biodiversidade do meio envolvente, mas também nos comportamentos e hábitos dos habitantes.

\section{Declaração de apoios financeiros}

A produção deste artigo inclui-se no plano de trabalhos do Projeto Exalar 2I, financiado pelo Fundo Europeu de
Desenvolvimento Regional (FEDER) e através da Fundação para a Ciência e Tecnologia, com referência PTDC/GES-AMB/30193/2017 (POCl-0I-0145-FEDER-030193, 02/ SAICT/20I7 - Project n. ${ }^{\circ}$ 30193). Ana Isabel Ribeiro foi financiada pela FCT com o contrato CEECIND/02386/2018.

\section{Conflito de interesses}

Os autores declaram que não existem conflitos de interesses.

\section{ORCID:}

Mariana Farraia (iD) 0000-0002-4959-0975

Inês Paciência (iD 0000-000I-5762-7814

Ana Isabel Ribeiro (iD) 0000-0001-8880-6962

André Moreira iD 0000-0002-7294-9296

Luís Delgado (iD) 0000-0003-2375-907।

João Cavaleiro Rufo (iD 0000-0003-|I75-242X

Contacto:

João Cavaleiro Rufo

Email: jcrufo@gmail.com

\section{REFERÊNCIAS}

I. Takano T, Nakamura K, Watanabe M. Urban residential environments and senior citizens' longevity in megacity areas: the importance of walkable green spaces. J Epidemiol Community Health 2002;56:913-8. doi: 10.1136/jech.56.12.913.

2. Tzoulas K, Korpela K, Venn S, Yli-Pelkonen V, Kaźmierczak A, Niemela J, et al. Promoting ecosystem and human health in urban areas using green infrastructure: A literature review. Landsc Urban Plan 2007;81:167-78.

3. de Vries S, Verheij RA, Groenewegen PP, Spreeuwenberg P. Natural environments - Healthy environments? An exploratory analysis of the relationship between greenspace and health. Environment and planning A: Economy and space 2003;35:1717-31.

4. Cilluffo G, Ferrante G, Fasola S, Montalbano L, Malizia V, Piscini $\mathrm{A}$, et al. Associations of greenness, greyness and air pollution exposure with children's health: a cross-sectional study in Southern Italy. Environ Health 2018;17:86. doi: 10.|I86/s 12940-0I8-0430-x.

5. Lepczyk CA, Aronson MFJ, Evans KL, Goddard MA, Lerman SB, Maclvor JS. Biodiversity in the City: Fundamental questions for understanding the ecology of urban green spaces for biodiversity conservation. BioScience 2017;67:799-807. 
6. Milligan C, Bingley A. Restorative places or scary spaces? The impact of woodland on the mental well-being of young adults. Health Place 2007;13:799-8II. doi: 10.1016/j.healthplace.2007.01.005.

7. Rufo JC, Paciência I, Ribeiro Al. Green environments and allergic diseases in children: A scoping review. Curr Epidemiol Reports 2019;6:442-8.

8. Aikins E, Ribeiro A. Elements of health and medical geography: Kendall hunt; 2020.

9. Landsat Science. Available from: https://landsat.gsfc.nasa.gov/.

10. Sentinel Online. Available from: https://sentinel.esa.int/web/sentinel/missions/sentinel-2.

II. Ribeiro Al, Krainski ET, Autran R, Teixeira H, Carvalho MS, de Pina MdF. The influence of socioeconomic, biogeophysical and built environment on old-age survival in a southern european city. Health Place 2016;41:100-109. doi: 10.1016/j.healthplace.2016. 08.008 .

12. Kondo MC, Fluehr JM, McKeon T, Branas CC. Urban green space and its impact on human health. Int J Environ Res Public Health 2018;15:445. doi: 10.3390/ijerphI5030445.

13. McCracken DS, Allen DA, Gow AJ. Associations between urban greenspace and health-related quality of life in children. Prev Med Rep 2016;3:21I-2I. doi: 10.1016/j.pmedr.2016.01.013.

14. Andrusaityte S, Grazuleviciene R, Kudzyte J, Bernotiene A, Dedele A, Nieuwenhuijsen MJ. Associations between neighbourhood greenness and asthma in preschool children in Kaunas, Lithuania: A case-control study. BMJ Open 2016;6:e0I034I. doi: 10.1136/ bmjopen-2015-010341

15. Dadvand P, Villanueva CM, Font-Ribera L, Martinez D, Basagaña $X$, Belmonte J, et al. Risks and benefits of green spaces for children: A cross-sectional study of associations with sedentary behavior, obesity, asthma, and allergy. Environ Health Perspect 2014; 122:1329-35. doi: 10.1289/ehp.1308038.

16. Donovan GH, Gatziolis D, Longley I, Douwes J. Vegetation diversity protects against childhood asthma: results from a large New Zealand birth cohort. Nat Plants 2018;4:358-364. doi: 10.1038/s41477-018-0151-8.

17. Fuertes E, Markevych I, Bowatte G, Gruzieva O, Gehring U, Becker A, et al. Residential greenness is differentially associated with childhood allergic rhinitis and aeroallergen sensitization in seven birth cohorts. Allergy 2016;71:I46I-7I. doi: I0.1III/all.12915.

18. Hsieh C-J, Yu P-Y, Tai C-J, Jan R-H, Wen T-H, Lin S-W, et al. Association between the first occurrence of asthma and residential greenness in children and teenagers in Taiwan. Int J Environ Res Public Health 2019;16:2076. doi: 10.3390/ijerph 16122076.

19. Tischer C, Gascon M, Fernández-Somoano A, Tardón A, Lertxundi Materola A, Ibarluzea J, et al. Urban green and grey space in relation to respiratory health in children. Eur Respir J 2017;49:15021/2. doi: 10.1183/13993003.02112-2015.
20. von Hertzen L, Hanski I, Haahtela T. Natural immunity: Biodiversity loss and inflammatory diseases are two global megatrends that might be related. EMBO Reports 2011;12:1089-93. doi: 10.1038/embor.2011.195.

21. von Mutius E. Biodiversity: The new kid on the block? J Allergy Clin Immunol 2018;141:I215-1216. doi: 10.1016/j.jaci.2018.01.036.

22. Rook GA. Regulation of the immune system by biodiversity from the natural environment: An ecosystem service essential to health. Proc Natl Acad Sci U S A 2013; I 10:18360-7. doi: 10.1073/pnas. I3I373III0..

23. Haahtela T. What is needed for allergic children? Pediatr Allergy Immunol 2014;25:2I-4. doi: 10.IIII/pai.I2I89.

24. Haahtela T, Holgate S, Pawankar R, Akdis CA, Benjaponpitak S, Caraballo $\mathrm{L}$, et al. The biodiversity hypothesis and allergic disease: World allergy organization position statement. World Allergy Organ J 2013;6:3. doi: 10.1186/1939-455I-6-3.

25. Escobedo FJ, Nowak DJ. Spatial heterogeneity and air pollution removal by an urban forest. Landsc Urban Plan 2009;90:102-10.

26. Chen E, Miller GE, Shalowitz MU, Story RE, Levine CS, Hayen R, et al. Difficult family relationships, residential greenspace, and childhood asthma. Pediatrics 2017;139:e20163056. doi: 10.1542/ peds.2016-3056.

27. Gernes R, Brokamp C, Rice GE, Wright JM, Kondo MC, Michael $\mathrm{YL}$, et al. Using high-resolution residential greenspace measures in an urban environment to assess risks of allergy outcomes in children. Sci Total Environ 2019;668:760-767. doi: 10.1016/j.scitotenv.2019.03.009.

28. Sa-Sousa A, Morais-Almeida M, Azevedo LF, Carvalho R, Jacinto T, Todo-Bom A, et al. Prevalence of asthma in Portugal - The portuguese national asthma survey. Clin Transl Allergy 20I2;2:15. doi: I0.1186/2045-7022-2-I5.

29. Morais-Almeida M, Santos N, Pereira AM, Branco-Ferreira M, Nunes C, Bousquet J, et al. Prevalence and classification of rhinitis in preschool children in Portugal: A nationwide study. Allergy 2013;68:1278-88. doi: 10.1III/all.12221.

30. Magalhães APTdF, Pina MdFRPd, Ramos EdCP. The role of urban environment, social and health determinants in the tracking of leisure-time physical activity throughout adolescence. J Adolesc Health 2017;60:100-106. doi: 10.1016/j.jadohealth.2016.08.015.

31. Pereira M, Nogueira H, Padez C. The role of urban design in childhood obesity: A case study in Lisbon, Portugal. Am J Hum Biol 2019;31:e23220. doi: 10.1002/ajhb.23220.

32. Paciência I, Cavaleiro Rufo J, Silva D, Martins C, Mendes F, Farraia $M$, et al. Exposure to indoor endocrine-disrupting chemicals and childhood asthma and obesity. Allergy 2019;74:1277-91. doi: I0.IIII/all.13740.

33. Ribeiro Al, Santos AC, Vieira VM, Barros H. Hotspots of childhood obesity in a large metropolitan area: Does neighbourhood social and built environment play a part? Int J Epidemiol 2020;49:934-943. doi: 10.1093/ije/dyz205. 
Carolina Dinis, Mariana Farraia, Inês Paciência, Ana Isabel Ribeiro, André Moreira, Luís Delgado, João Cavaleiro Rufo

34. Paciência I, Rufo JC, Silva D, Martins C, Mendes F, Rama T, et al. School environment associates with lung function and autonomic nervous system activity in children: a cross-sectional study. Sci Rep 2019;9:15156. doi: 10.1038/s41598-019-51659-y.

35. Ribeiro Al, Tavares C, Guttentag A, Barros H. Association between neighbourhood green space and biological markers in school-aged children. Findings from the Generation XXI birth cohort. Environ Int 2019;132:105070. doi: 10.1016/j.envint.2019.105070
36. Cavaleiro Rufo J, Paciência I, Hoffimann E, Moreira A, Barros H, Ribeiro Al. The neighbourhood natural environment is associated with asthma in children: A birth cohort study. Allergy 2021;76: 348-358. doi: 10.1III/all.14493.

37. Paciência I, Cavaleiro Rufo J, Ribeiro Al, Mendes FC, Farraia M, Silva $D$, et al. Association between the density and type of trees around urban schools and exhaled nitric oxide levels in schoolchildren. Eur Ann Allergy Clin Immunol 2021;53:29-36. doi: 10.23822/EurAnnACI.1764-1489.162. 